\title{
Return, Volatility and Shock Spillovers of Bitcoin with Energy and Technology Companies *
}

\author{
Efthymia Symitsi and Konstantinos J. Chalvatzis \\ Norwich Business School, University of East Anglia
}

\begin{abstract}
We employ an asymmetric multivariate VAR-GARCH model to study spillover effects between Bitcoin and energy and technology companies. We find unilateral return and volatility spillovers and bidirectional shock influences and demonstrate portfolio management implications of dynamic conditional correlations.
\end{abstract}

Keywords: Bitcoin, Energy, Technology, Spillovers, Multivariate GARCH

JEL: C22, C3, C5, G1, G11

*Corresponding author is Efthymia Symitsi. Tel.: +44 (0)1603 59 7241; Email address: E.Symitsi@uea.ac.uk. 


\section{Introduction}

Cryptocurrencies have received significant attention from investors, media and regulatory authorities with a burgeoning academic interest from computer science to finance literature (Böhme et al. 2015). Despite the huge number of cryptocurrencies, Bitcoin maintains the lion's share with substantial market capitalization. Bitcoins are backed with blockchain technology which allows a decentralized system for the introduction of new Bitcoins and verification of transactions by solving a crypto-puzzle. The requirements in terms of computing power and energy are enormous as Bitcoin transactions increase, more miners compete in the Bitcoin network, and the crypto-algorithm that verifies blocks and rewards miners becomes more difficult. The total annual energy consumption amounts to 57.69 TWh, close to the electricity needs of Kuwait (BitcoinEnergyConsumption.com, March 2018). Despite the strong interdependence between energy, technology and Bitcoin, their dynamics and economic linkages have not yet been explored.

This study fills the gap by contributing in two ways. First, we use a Vector Autoregression conditional mean process to model returns and the asymmetric BEKK Generalized Autoregressive Conditional Heteroskedasticity process for variances (VAR-BEKKAGARCH) to examine return, volatility, and shock spillovers between Bitcoin and stock indices of clean energy, fossil fuel energy and technology companies 11 Second, we study portfolio management implications of dynamic conditional correlations in a minimumvariance optimal portfolio.

Our study expands previous efforts in cryptocurrencies' literature that analyze the diversification benefits and interdependencies with financial assets (Dyhrberg, 2016; Ciaian et al., 2018; Corbet et al., 2018) and explore Bitcoin returns and volatility (Balcilar et al., 2017; Katsiampa, 2017). Our work is closely related to the strand that investigates spillovers in energy and technology firms (Sadorsky, 2012) and studies that connect Bitcoin with energy prices, the key element for its production and sustainability (Bouri

${ }^{1}$ The supply of low-cost energy plays an important role in the high energy demands of Bitcoin miners. For instance, the abundance of geothermal and hydroelectric energy in Iceland attracts Bitcoin miners consuming more power than households (https://grist.org/article/bitcoin-gobblesup-clean-energy-just-when-the-real-world-needs-it-most/). Recent renewable energy PPA contracts have lowered prices dramatically and are in a trajectory to continue doing so in the next years (https://cms.irena.org/publications/2018/Jan/Renewable-power-generation-costs-in-2017). Pricing for new projects is so favourable for renewables that worldwide in 2017 more solar was installed than any other fossil fuel energy source (https://www.unenvironment.org/news-and-stories/press-release/bankingsunshine-world-added-far-more-solar-fossil-fuel-power). 
et al., 2017; Hayes, 2017).

Our paper is organized as follows. Section 2 presents the data and methodology. Section 3 discusses the empirical findings and Section 4 provides the main conclusions.

\section{Data and Methodology}

We obtain data for S\&P Global Clean Energy Index (SPGCE), MSCI World Energy Index (MSCIWE)2, MSCI World Information Technology Index (MSCIWIT) and Bitcoin (BTC) from Datastream spanning from August 22, 2011 to February 15, 2018 . $^{3}$ Our sample corresponds to a total of 1,696 daily observations. The returns are computed as the first difference of the natural logarithm of prices multiplied by 100 .

Table 1. Descriptive Statistics of Daily Returns

\begin{tabular}{|c|c|c|c|c|}
\hline & SPGCE & MSCIWE & MSCIWIT & BTC \\
\hline Mean & -0.0156 & -0.0034 & 0.0633 & 0.4026 \\
\hline StDev & 1.2509 & 1.1803 & 0.9138 & 6.1824 \\
\hline Min & -6.283 & -6.058 & -4.5662 & -66.3948 \\
\hline Max & 7.0593 & 5.0688 & 4.5613 & 48.4776 \\
\hline Skewness & -0.2468 & -0.146 & -0.2982 & -1.0339 \\
\hline Kurtosis & 2.8313 & 2.5515 & 2.5907 & 19.8119 \\
\hline \multicolumn{5}{|c|}{ Correlation Matrix } \\
\hline MSCIWE & $0.5914^{* * *}$ & & & \\
\hline MSCIIT & $0.6087^{* * *}$ & $0.6288^{* * *}$ & & \\
\hline BTC & $0.0417^{*}$ & 0.0227 & 0.0318 & \\
\hline
\end{tabular}

Table 1 displays the summary statistics and the correlation matrix. The unconditional correlation of BTC with SPGCE is marginally significant, while the correlations between stock indices are positive and strong. Figure 1 shows the time series of prices. BTC has remarkable returns which are accompanied with extreme volatility that is five times higher than the most volatile stock index.

Our study employs the VAR(1)-BEKK-AGARCH model of McAleer et al. (2009) which considers asymmetries of negative shocks on conditional variance. 4 The conditional mean and variance are described as follows:

\footnotetext{
${ }^{2}$ MSCIWE contains only firms related to fossil fuel energy.

${ }^{3}$ The analysis is replicated to the period $22 / 08 / 2011-31 / 12 / 2017$ to avoid a possible structural change due to the plummet in Bitcoin prices. The results remain unchanged and are available upon request.

${ }^{4}$ The number of autoregressive terms for the VAR model is selected by the AIC lag order criterion.
} 


$$
\begin{aligned}
R_{t} & =C+\Phi R_{t-1}+\varepsilon_{t}, \quad \varepsilon_{t} \mid F_{t-1} \sim N\left(0, H_{t}\right) \\
\varepsilon_{t} & =z_{t} \sqrt{H_{t}}, z_{t} \sim N(0,1) \\
H_{t} & =\Psi^{\prime} \Psi+A^{\prime} \varepsilon_{t-1} \varepsilon_{t-1}^{\prime} A+B^{\prime} H_{t-1} B+\Delta^{\prime} I_{t-1} \varepsilon_{t-1} \varepsilon_{t-1}^{\prime} \Delta,
\end{aligned}
$$

where $R_{t}$ is a vector of returns on clean and fossil fuel energy, technology, and Bitcoin $(i=1,2,3,4)$ at time $t ; \varepsilon_{t}$ is the error term; $z_{t}$ is an i.i.d. process and $H_{t}$ is the conditional variance-covariance matrix. The past information available at time $t-1$ is denoted as $F_{t-1}$. The model parameters of the multivariate GARCH specification $(C, \Phi, \Psi, A, B$, $\Delta)$ are estimated by Quasi-Maximum-Likelihood using the BFGS algorithm and robust standard errors.
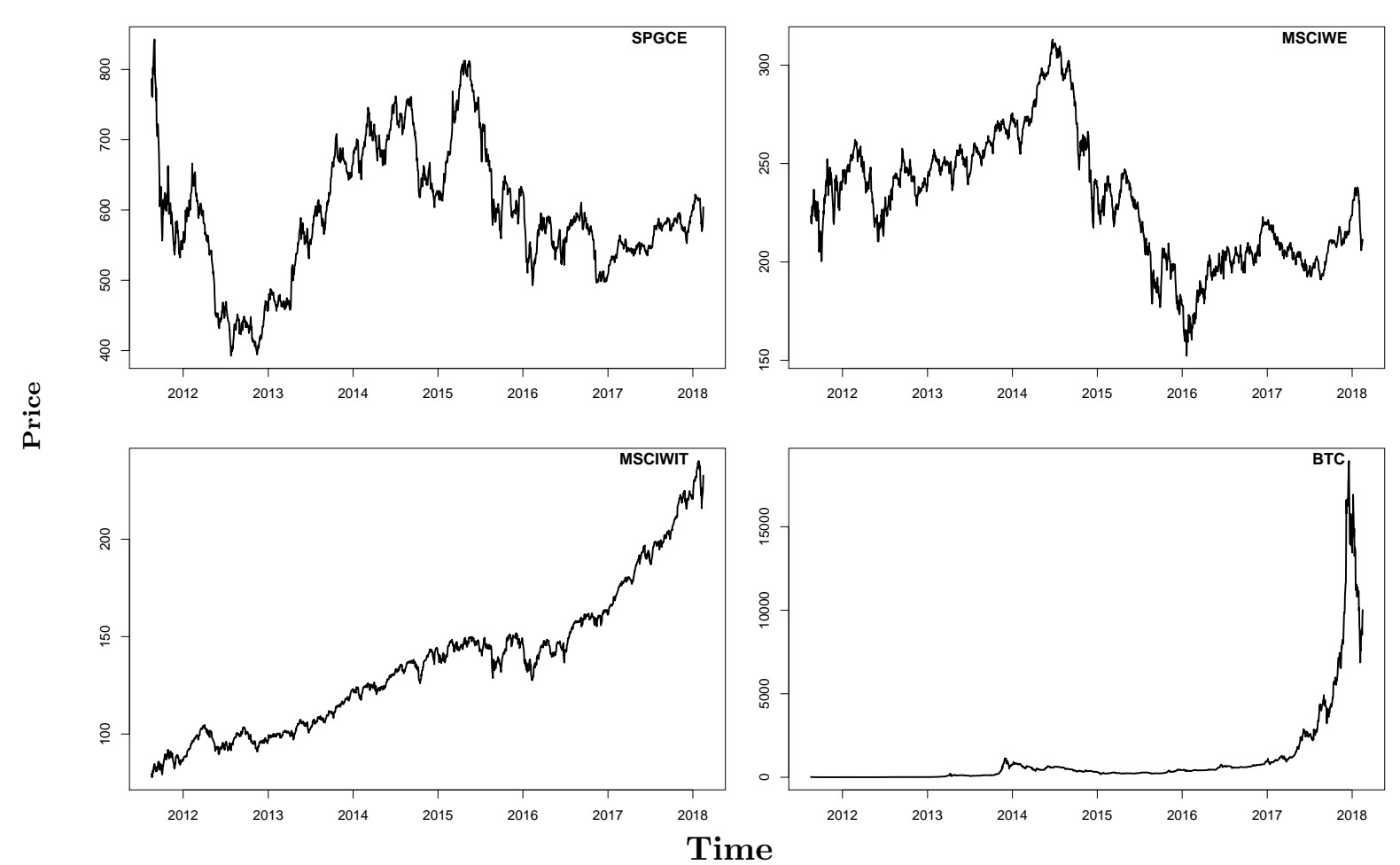

Figure 1. Daily Prices 


\section{Empirical Results}

\subsection{Return, Volatility and Asymmetric Shock Spillovers}

The estimation results are presented in Table 2. We find significant and positive past own return effects on energy indices $\left(\phi_{11}, \phi_{22}\right)$. Past one-period lagged returns of Bitcoin and technology firms do not help predict short-term returns. The $\phi_{41}, \phi_{42}$ and $\phi_{43}$ parameters in VAR-mean equation reveal unilateral past return spillovers from stock indices to Bitcoin. In other words, higher returns in clean energy companies predict lower returns in Bitcoin, while there is a positive impact of fossil fuel energy and information technology past returns on Bitcoin.

These relationships are explained by the mining process where miners are rewarded with Bitcoins and become participants in cryptocurrency markets. Since energy and technology are key inputs in Bitcoin production, they can determine the required returns of miners. Fossil fuel energy and information technology firms are better off when there is stability in the market and there are prospects for development and higher future cash flows. Thus, more expensive new technology and energy prices put similar pressures on Bitcoin prices. Conversely, the period under investigation while producers of clean energy were benefited by government subsidies and fixed-term contracts, clean energy distributors offered reduced prices when the production was abundant without opportunities for energy storage or change in the production. The finite Bitcoin mining, the higher energy efficiency with storage solutions and the smaller and cheaper equipment are expected to change these relationships in the future.

As for the estimates of variance-covariance equations, own conditional $\mathrm{ARCH}\left(\alpha_{i i}\right)$ and GARCH $\left(\beta_{i i}\right)$ effects are indicative of short and long-run persistence, respectively. Our findings suggest stronger and larger long-run persistence of own volatility than shortterm persistence. MSCIWE exhibits the highest long-run persistence, followed by BTC, SPGCE, and MSCIWIT. Short-term volatility spills over from technology companies to Bitcoin $\left(\alpha_{43}\right)$ that could be explained by the increasing demand of miners for advanced high-technology products from computer hardware manufacturing companies. Unilateral long-term spillovers from Bitcoin to fossil fuel and clean energy stocks $\left(\beta_{14}, \beta_{24}\right)$ are indicative of the effect of Bitcoin on energy demand in the long-run. We also find evidence of bilateral negative shock or "bad news" transmissions between Bitcoin and fossil fuel 
Table 2. VAR(1)-BEKK-AGARCH Parameter Estimates

\begin{tabular}{|c|c|c|c|c|c|}
\hline \multirow[t]{2}{*}{ Variable } & Mean & \multicolumn{4}{|c|}{ Variance } \\
\hline & $\phi$ & $\psi$ & $\alpha$ & $\boldsymbol{\beta}$ & $\delta$ \\
\hline$(1,0)$ & \multicolumn{5}{|l|}{0.0009} \\
\hline$(1,1)$ & $0.0700^{* * *}$ & $0.2464^{* * *}$ & $0.2400^{* * *}$ & $0.9259^{* * *}$ & $0.2163^{* * *}$ \\
\hline$(1,2)$ & $0.0849^{* * *}$ & & 0.0311 & 0.0381 & -0.0069 \\
\hline$(1,3)$ & $0.1191^{* * *}$ & & 0.0032 & 0.0639 & -0.0352 \\
\hline$(1,4)$ & 0.0043 & & 0.3461 & $-0.2791^{* * *}$ & 0.2317 \\
\hline$(2,0)$ & \multicolumn{5}{|l|}{0.0067} \\
\hline$(2,1)$ & -0.0222 & -0.0294 & -0.0035 & $0.0266^{* *}$ & -0.0240 \\
\hline$(2,2)$ & $0.0784^{* * *}$ & $0.1234^{* * *}$ & 0.0175 & $0.9591^{* * *}$ & $0.3792^{* * *}$ \\
\hline$(2,3)$ & 0.0064 & & $0.0751^{*}$ & $-0.0240^{*}$ & $0.1245^{* * *}$ \\
\hline$(2,4)$ & 0.0006 & & -0.0129 & $0.1575^{* * *}$ & $-0.4639^{* * *}$ \\
\hline$(3,0)$ & \multicolumn{5}{|l|}{$0.0731^{* * *}$} \\
\hline$(3,1)$ & -0.0002 & -0.0153 & $-0.0871^{* *}$ & -0.0190 & $0.1365^{* *}$ \\
\hline$(3,2)$ & -0.0019 & $0.2029^{* * *}$ & -0.0881 & $-0.0563^{*}$ & 0.0099 \\
\hline$(3,3)$ & 0.0346 & $0.1182^{*}$ & $-0.1067^{*}$ & $0.8742^{* * *}$ & $0.3474^{* * *}$ \\
\hline$(3,4)$ & \multirow{2}{*}{\multicolumn{5}{|c|}{$0.2565^{* * *}$}} \\
\hline$(4,0)$ & & & & & \\
\hline$(4,1)$ & $-0.3043^{* * *}$ & $0.3157^{* *}$ & 0.0053 & -0.0005 & 0.0040 \\
\hline$(4,2)$ & $0.1787^{* *}$ & -0.0052 & -0.0022 & 0.0001 & $-0.0110^{* *}$ \\
\hline$(4,3)$ & $0.2245^{* * *}$ & 0.1074 & $-0.0053^{* *}$ & 0.0013 & $-0.0100^{*}$ \\
\hline$(4,4)$ & 0.0006 & -0.0000 & $0.3795^{* * *}$ & $0.9326^{* * *}$ & -0.0975 \\
\hline Information Criteria & & \multicolumn{4}{|c|}{ Diagnostics of standardized $\varepsilon_{t}$ and $\varepsilon_{t}^{2}$} \\
\hline $\log L$ & -11320.132 & $\mathrm{Q}(4)$ & \multicolumn{3}{|c|}{$77.985[0.112]$} \\
\hline AIC & 13.457 & $\mathrm{Q}(20)$ & \multicolumn{3}{|c|}{$394.203[0.003]$} \\
\hline SBC & 13.707 & $\mathrm{Q}^{2}(4)$ & \multicolumn{3}{|c|}{$5.104[0.277]$} \\
\hline HQ & 13.550 & $\mathrm{Q}^{2}(20)$ & \multicolumn{3}{|c|}{$15.559[0.741]$} \\
\hline
\end{tabular}

Note. $* * *, * *, *$ denote significance at $1 \%, 5 \%$, and $10 \%$, respectively. P-values for the squared standardized residual diagnostics are reported in brackets.

energy and technology companies $\left(\delta_{24}, \delta_{34}, \delta_{42}, \delta_{43}\right)$.

Even though we do not employ in our main analysis DCC or CCC alternatives of McAleer et al. (2009) due to their deficiencies in capturing cross market spillovers, we compare the VAR(1)-BEKK-AGARCH fit against them to increase the robustness of our results.5 LogLikelihood, AIC, SBC and Hannan-Quinn criteria indicate that our model best captures the spillovers and dynamics of volatilities, shocks and returns for Bitcoin and energy and technology stock indices. Table 2 also presents diagnostics for standardized and squared standardized residuals. The multivariate Ljung-Box tests indicate that the model is adequate failing to find residual independence at conventional significance levels for large number of lags.

\subsection{Dynamic Correlations and Portfolio Management Implica- tions}

The strong variations of conditional correlations suggest that the assumption of constant dependencies is not realistic. Additionally, the low correlation of Bitcoin with stock

\footnotetext{
${ }^{5}$ To conserve space, we do not tabulate the parameters of DCC and CCC spillover models. The results are available upon request.
} 
indices demonstrates its potential as an investment opportunity.

Table 3. Minimum-Variance Portfolios

\begin{tabular}{|c|c|c|c|c|}
\hline & SPGCE & MSCIWE & MSCIWIT & ВTC \\
\hline \multicolumn{5}{|l|}{ Weights } \\
\hline$\overline{\text { Mean }}$ & 0.1408 & 0.2461 & 0.5746 & 0.0384 \\
\hline StDev & 0.1390 & 0.2069 & 0.2075 & 0.0369 \\
\hline Max & 0.9161 & 0.8821 & 1.0000 & 0.2485 \\
\hline Investment & 0.8111 & 0.8200 & 0.9911 & 0.9392 \\
\hline Portfolio $\mathbf{R}$ & \multicolumn{4}{|c|}{0.0827} \\
\hline Portfolio $\mathbf{R}$ & \multicolumn{4}{|c|}{0.7965} \\
\hline
\end{tabular}

To illustrate the implications of our findings for a risk averse investor who invests in these assets, we estimate optimal weights for the global minimum-variance portfolio which minimizes the risk without reducing the expected returns. This strategy requires only the variance-covariance matrix and deals with concerns for the large volatility of Bitcoin exploiting the benefits from its low correlations with other assets. Moreover, since weights are disentangled from asset returns, our findings are not influenced by extreme Bitcoin prices or bubble periods. The global minimum-variance portfolio solves the following problem in each period $t$ :

$$
\min w_{t}^{\prime} H_{t} w_{t} \quad \text { s.t. } \quad w_{t}^{\prime} \iota=1 \quad \text { and } \quad w_{t} \geq 0 \text {, }
$$

where $w_{t}$ is an $4 \times 1$ vector of portfolio weights and $\iota$ is an $4 \times 1$ vector of ones. The constraints ensure that the sum of portfolio weights should be equal to one and short-sales are not allowed. The optimal portfolio weights are given by:

$$
w_{t}=H_{t}^{-1} \iota / \iota^{\prime} H_{t}^{-1} \iota
$$

Table 3 presents the summary statistics of portfolio weights for each asset along with the participation of each asset in the portfolios across the total sample period (Investment). Bitcoin contributes to 1,591 out of 1,694 trading periods (93.92\%) maintaining a small average weight of 3.84\%. Our findings indicate that the low correlation of Bitcoin with the stock indices trades off the large variance and leads to higher returns $(8.27 \%)$ and lower portfolio risk $(79.65 \%)$ in comparison to a portfolio that does not include Bitcoin (4.38\% and $89.40 \%$, respectively). 


\section{Conclusions}

This study uses a VAR(1)-AGARCH model to analyze spillover effects between Bitcoin and energy and technology companies. Our findings indicate significant return spillovers from energy and technology stocks to Bitcoin. Short-run volatility spills over from technology companies to Bitcoin, while Bitcoin has long-run volatility effects to energy companies. We find bidirectional asymmetric shock spillovers between Bitcoin and stock indices. Finally, we show portfolio management implications and benefits from the low dependence of Bitcoin with the stock indices.

\section{References}

Balcilar, M., Bouri, E., Gupta, R. and Roubaud, D. (2017), 'Can volume predict Bitcoin returns and volatility? A quantiles-based approach', Economic Modelling 64, 74-81.

Böhme, R., Christin, N., Edelman, B. and Moore, T. (2015), 'Bitcoin: Economics, Technology, and Governance', Journal of Economic Perspectives 29(2), 213-38.

Bouri, E., Jalkh, N., Molnár, P. and Roubaud, D. (2017), 'Bitcoin for energy commodities before and after the December 2013 crash: diversifier, hedge or safe haven?', Applied Economics 49(50), 5063-5073.

Ciaian, P., Rajcaniova, M. and Kancs, d. (2018), 'Virtual relationships: Short-and longrun evidence from BitCoin and altcoin markets', Journal of International Financial Markets, Institutions and Money 52, 173-195.

Corbet, S., Meegan, A., Larkin, C., Lucey, B. and Yarovaya, L. (2018), 'Exploring the dynamic relationships between cryptocurrencies and other financial assets', Economics Letters 165, 28-34.

Dyhrberg, A. H. (2016), 'Bitcoin, gold and the dollar-A GARCH volatility analysis', Finance Research Letters 16, 85-92.

Hayes, A. S. (2017), 'Cryptocurrency value formation: An empirical study leading to a cost of production model for valuing bitcoin', Telematics and Informatics 34(7), 13081321. 
Katsiampa, P. (2017), 'Volatility estimation for Bitcoin: A comparison of GARCH models', Economics Letters 158, 3-6.

McAleer, M., Hoti, S. and Chan, F. (2009), 'Structure and Asymptotic Theory for Multivariate Asymmetric Conditional Volatility', Econometric Reviews 28(5), 422-440.

Sadorsky, P. (2012), 'Correlations and volatility spillovers between oil prices and the stock prices of clean energy and technology companies', Energy Economics 34(1), 248-255. 